\title{
Associação dos fatores de risco para doenças cardiovasculares e qualidade de vida entre servidores da saúde
}

CDD. 20.ed. 306

613.7

\author{
Matheus BAREL* \\ Juliana Cavalcante de Andrade LOUZADA* \\ Henrique Luiz MONTEIRO* \\ Sandra Lia do AMARAL*
}

*Faculdade de Ciências, Universidade Estadual Paulista - Bauru.

\section{Resumo}

Inúmeros problemas de saúde pública são atribuidos aos fatores induzidos pelo ambiente de trabalho que, associados ao estilo de vida sedentário, contribuem para deteriorar a qualidade de vida do trabalhador. Neste sentido, procuramos descrever o perfil, avaliar a condição física e o risco cardiovasculares dos funcionários da saúde. Foram avaliados 198 funcionários (46 \pm 10 anos) que responderam uma anamnese e foram submetidos a avaliações bioquímicas, antropométricas e funcionais. Os valores foram apresentados como média \pm desvio padrão da média. Foram utilizados testes de qui-quadrado $\left(\mathrm{x}^{2}\right)$, taxa de "odds" e correlações de Pearson $(p<0,05)$. Dos avaliados, 54\% apresentaram peso corporal elevado, 33\% pressão arterial (PA) elevada e $56 \%$ eram sedentários. Colesterol total (CT), lipoproteína de baixa densidade e triglicerídeos (TG) acima da normalidade foram encontrados em 49\%, 41\% e 24\% dos servidores, respectivamente e, $75 \%$ apresentaram taxas reduzidas de lipoproteína de alta densidade. As análises de $\mathrm{x}^{2} \mathrm{e}$ taxa de "odds" apontaram que os valores de TG e PA foram significativamente maiores para homens comparados às mulheres. Capacidade cardiorrespiratória ruim foi verificada em 56\% dos homens e 23\% das mulheres, além disso, a flexibilidade apresentou-se com níveis insatisfatórios para homens e mulheres. Observou-se que o índice de massa corporal se correlacionou positivamente com PA, CT e TG. Constatou-se, também que a idade foi fator agravante para PA, flexibilidade, CT e glicemia nas mulheres. Dessa forma, a prevalência de múltiplos fatores de risco nestes trabalhadores demonstra tratar-se de população não assistida pela saúde, motivo pelo qual sugere-se conscientização sobre sua própria saúde evitando a aposentadoria precoce.

UnITERMos: Hipertensão; Diabetes; Sedentarismo; Obesidade.

\section{Introdução}

Atualmente as doenças do aparelho circulatório são responsáveis pelos maiores índices de mortalidade e morbidade (STUMer, HasselbaCh \& AMELANG, 2006). Hipertensão arterial, obesidade, tabagismo, diabetes tipo 2 e sedentarismo contribuem isolada ou associadamente para o desenvolvimento do processo aterosclerótico, que caracteriza-se como mecanismo central para o desenvolvimento de doença arterial coronariana (Thompson, Buchner, Pina, Balady, Williams, Marcus, Berra, Blair, Costa, Franklin, Fletcher, Gordon, Pate, Rodriguez, Yancey \& Wenger, 2003), responsável por 28\% dos óbitos no Brasil (Brasil, 2009). Na cidade de Bauru e Jaú, no Estado de São Paulo, estes agravos levam a óbito
$34 \%$ e $32 \%$ da população, respectivamente. Neste panorama, as doenças infecto-contagiosas gradativamente têm deixado de ser o principal foco de atenção em saúde, cedendo espaço para afecções relacionadas ao ambiente de trabalho, o qual, juntamente com outros fatores de risco, vêm se destacando como um dos determinantes de doenças crônico-degenerativas.

Tem sido demonstrado que muitos problemas de saúde pública são atribuídos aos fatores induzidos pelo ambiente de trabalho que, associados ao estilo de vida sedentário, contribuem para deteriorar a qualidade de vida do trabalhador (GUS, HARZHEIM, ZASLAVSKY, MEdina \& Gus, 2004; Matos, Silva, Pimenta \& CunHa, 2004; Rosini, Machado \& Xavier, 2006; Valles, 
Mate, Bronsoms, Campins, Rosello, Torquet \& Mauri, 1997; Viebig, Valero, Araújo, Yamada \& Mansur, 2006). Os avanços tecnológicos, a forte pressão psicológica, pouco tempo de lazer, atividades ocupacionais excessivas, baixos salários e dificuldades de acesso à assistência médica são considerados potenciais fatores de risco para o desenvolvimento de doenças cardiovasculares (HWANG, TSAI \& CHEN, 2006).

Embora muitos estudos já tenham comprovado os benefícios do exercício físico regular, há uma expressiva parcela da população que é sedentária. Entre os brasileiros, $80,3 \%$ não praticam exercícios habitualmente e, na região sudeste, esta proporção atinge 78,2\% dos adultos (Sociedade Brasileira de Cardiologia, 2005).

\section{Métodos}

\section{Amostra}

A casuística do estudo foi constituída por 198 funcionários da Divisão Regional de Saúde - VI (DRSVI) e do NGA-25 (Núcleo de Gestão Assistencial-25) de Jaú. Todos os participantes foram voluntários. Somente foram excluídos do estudo aqueles que tinham contra-indicação médica em sua avaliação inicial. Todos os participantes leram e assinaram um termo de consentimento livre e esclarecido.

\section{Procedimentos}

Todos os procedimentos foram aprovados pelo Comitê de Ética da Secretaria da Saúde de Bauru. Primeiramente os dados foram coletados por meio de entrevista dirigida que continha informações sobre sexo, idade, histórico de doenças e medicamentos utilizados e intervenções cirúrgicas. Além disso, foram coletadas informaçôes sobre grau de escolaridade, bens e serviços que os servidores tinham acesso, para a determinação do estrato social a que pertencem.

Todos os indivíduos foram submetidos à avaliação médica e aos seguintes exames laboratoriais: Colesterol Total (CT), Lipoproteína de Alta Densidade (HDL-C), Lipoproteína de Baixa Densidade (LDL-C), Triglicerídeos (TG) e Glicemia de jejum (GLI), realizados nos Hospitais Conveniados de cada município. A classificação dos valores do perfil lipídico foi determinada de acordo com as IV Diretrizes Brasileiras de Dislipidemia e Prevenção da Aterosclerose (Sociedade Brasileira de CardioLOGIA, 2007).
Várias iniciativas têm sido efetuadas para sensibilizar e conscientizar a população a adotar hábitos regulares de exercícios físicos, no entanto, a maioria das campanhas é muito pontual (um dia) e por isso causam poucos impactos positivos sobre a atitude das pessoas.

Complementarmente às estratégias em Saúde Pública para sensibilizar a população a adotar hábitos saudáveis, é importante conhecer a prevalência dos fatores riscos cardiovasculares isolados ou associados em grupos sociais e/ou ocupacionais específicos.

Por esse motivo, os objetivos da presente investigação foram descrever o perfil, avaliar a condição física e o risco cardiovascular dos funcionários da saúde.

Massa corporal $(\mathrm{kg})$ e estatura $(\mathrm{cm})$ foram medidos com uma balança (Filizola) a partir dos quais se calculou o Índice de Massa Corporal (IMC), sendo este classificado da seguinte forma: IMC entre 25,1 $\mathrm{kg} / \mathrm{m}^{2}$ e $29,9 \mathrm{~kg} / \mathrm{m}^{2}$ - sobrepeso e IMC maior que 30 $\mathrm{kg} / \mathrm{m}^{2}$ - obesidade. A flexibilidade foi avaliada por meio do teste de sentar-e-alcançar e os resultados foram classificados em baixo (maior que $30 \mathrm{~cm}$ e $24 \mathrm{~cm}$ para mulheres e homens, respectivamente), médio (entre 30 e $33 \mathrm{~cm}$ e entre 24 e $28 \mathrm{~cm}$ para mulheres e homens, respectivamente e elevado (acima de 33 e $28 \mathrm{~cm}$ para mulheres e homens, respectivamente) (Pollock \& Wilmore, 1993).

A Pressão Arterial (PA) foi aferida por esfigmomanômetro com aneróide (Solidor) após 10 minutos de repouso. Os valores foram obtidos com uma média de três aferições consecutivas (SOCIEDADE BrasiLEIRA de Cardiologia. Sociedade Brasileira de HipertenSÃo. Sociedade Brasileira de Nefrologia, 2007) na posição sentada; os valores acima de $140 \mathrm{mmHg}$ para PA sistólica e $90 \mathrm{~mm} \mathrm{Hg}$ para PA diastólica foram considerados elevados.

Para avaliar a capacidade funcional (PoLlock \& WILMORE, 1993), foi realizada avaliação ergométrica em esteira rolante, utilizando o protocolo de Ellestad modificado. Os critérios utilizados para encerrar o teste foram: atingir frequência sub-máxima de $85 \%$ da frequência cardíaca para normotensos e $75 \%$ para hipertensos, elevação abrupta da pressão arterial ou redução persistente da frequência cardíaca, cansaço físico intenso, dores ou dispnéia. $\mathrm{O}$ resultado do teste permitiu o cálculo do consumo máximo de oxigênio baseado em modelo matemático específico, 
e este consumo foi classificado em baixo $(<26 \mathrm{ml} /$ $\mathrm{kg} / \mathrm{min}$ e $<37 \mathrm{ml} / \mathrm{kg} / \mathrm{min}$ para mulheres e homens, respectivamente), médio (entre 26 e $31 \mathrm{ml} / \mathrm{kg} / \mathrm{min}$ e 37 e $39 \mathrm{ml} / \mathrm{kg} / \mathrm{min}$ para mulheres e homens, respectivamente) e elevado (acima de $31 \mathrm{ml} / \mathrm{kg} / \mathrm{min}$ e $39 \mathrm{ml} /$ $\mathrm{kg} / \mathrm{min}$ para homens e mulheres, respectivamente).

$\mathrm{O}$ risco cardiovascular de cada funcionário foi estimado a partir do protocolo proposto pela American Heart Association (KaVEY, Daniels, LAUER, Atkins, Hayman \& Taubert, 2003).

\section{Análise estatística}

Os resultados foram organizados sob a forma de média \pm desvio padrão da média. As respostas dos questionários foram apresentadas sob a forma de distribuição de frequência relativa percentual. As

\section{Resultados}

Dos 198 funcionários da saúde avaliados, 155 eram mulheres (78\%) e 43 homens (22\%) com média de idade de $46 \pm 10$ anos. A classe social predominante foi a B (47\%), seguida pela C (37\%), D $(6,5 \%)$, A (2\%), E (0,5\%). Apenas 35\% dos trabalhadores possuíam ensino superior completo, 32\% ensino médio completo, $10 \%$ ensino fundamental, $11 \%$ primário completo e $4 \%$ eram analfabetos.

A FIGURA 1 apresenta a distribuição de frequência dos principais fatores de risco encontrados nesta população. As taxas representam a somatória dos valores limítrofes com aqueles que superam esta faixa para cada valor isolado, corrigido por diferenças quanto a sexo e idade. A condição de obesidade (IMC $>30 \mathrm{~kg} / \mathrm{m}^{2}$ ) foi observada em 23\% dos funcionários, enquanto $31 \%$ apresentavam sobrepeso (IMC 25-30 $\mathrm{kg} / \mathrm{m}^{2}$ ), resultando em $54 \%$ dos servidores acima do peso ideal. O tabagismo foi referido por 33\% das pessoas, sendo 17\% fumantes e 16\% ex-fumantes. Em relação ao perfil lipídico, valores de colesterol total elevados foram observados em $49 \%$ dos servidores $(16 \%>240 \mathrm{mg} / \mathrm{dL})$. Da mesma forma, $41 \%$ apresentaram taxas de LDL-C alteradas (13\% $>160 \mathrm{mg} / \mathrm{dL}$ ). Triglicerídeos elevado foi encontrado em $24 \%$ dos casos $(12 \%>200 \mathrm{mg} / \mathrm{dL})$. Valores reduzidos de HDL-C foram detectados em $75 \%$ análises estatísticas utilizadas foram: 1) distribuição de qui-quadrado para comparação da variável de interesse segundo respectiva classificação comparando sexo; 2) a correlação de Pearson do IMC e da idade com as variáveis hemodinâmicas, bioquímicas e antropométricas, sendo descritos apenas os casos onde o "p-value" de um dos sexos ou ambos mostrou-se significativo; 3) a taxa de "odds" foi adotada para comparação de sexo considerando as mulheres como padrão de referência, por conta de serem estas as que acessam mais os serviços de saúde e, portanto, têm maior chance de diagnóstico precoce e tratamento adequado, condição importante para o caso de doenças crônicas. Destarte a referida taxa foi utilizada para observar o risco a que os homens estão expostos de terem precarizadas as condições de saúde. $\mathrm{O}$ nível de significância adotado foi de $\mathrm{p}<0,05$.

dos funcionários $(18 \%<40 \mathrm{mg} / \mathrm{dL})$. A glicemia de jejum mostrou-se alterada em $10 \%$ dos indivíduos $(4 \%>126 \mathrm{mg} / \mathrm{dL})$. Valores elevados de pressão arterial (>140 $\mathrm{mmHg}$ para PAS e >90 $\mathrm{mmHg}$ para PAD) no dia da avaliação foram observados em 33\% dos servidores e $74 \%$ apresentaram risco coronariano oscilando entre baixo a elevado. A condição de sedentarismo foi o terceiro fator de risco coronariano mais encontrado, acometendo $56 \%$ dos funcionários.

Os diversos fatores de risco analisados anteriormente (FIGURA 1) foram, ainda, avaliados separadamente por sexo. As frequências das variáveis analisadas por meio da estatística de qui-quadrado estão descritas na TABELA 1. Somente 30\% dos fatores de risco analisados apresentam diferenças entre os sexos. Os valores de HDL-C, triglicerídeos e pressão arterial estavam significativamente maiores para os homens quando comparados às mulheres. As demais variáveis não expressaram diferenças estatísticas significativas. Como os fatores de risco têm comportamento distinto entre os sexos, a taxa de "odds" foi utilizada para melhor avaliar tais diferenças. Os resultados da taxa de "odds" (TABELA 2) apontaram que somente os valores de triglicerídeos e pressão arterial foram significativamente superiores para o sexo masculino comparativamente ao feminino. 


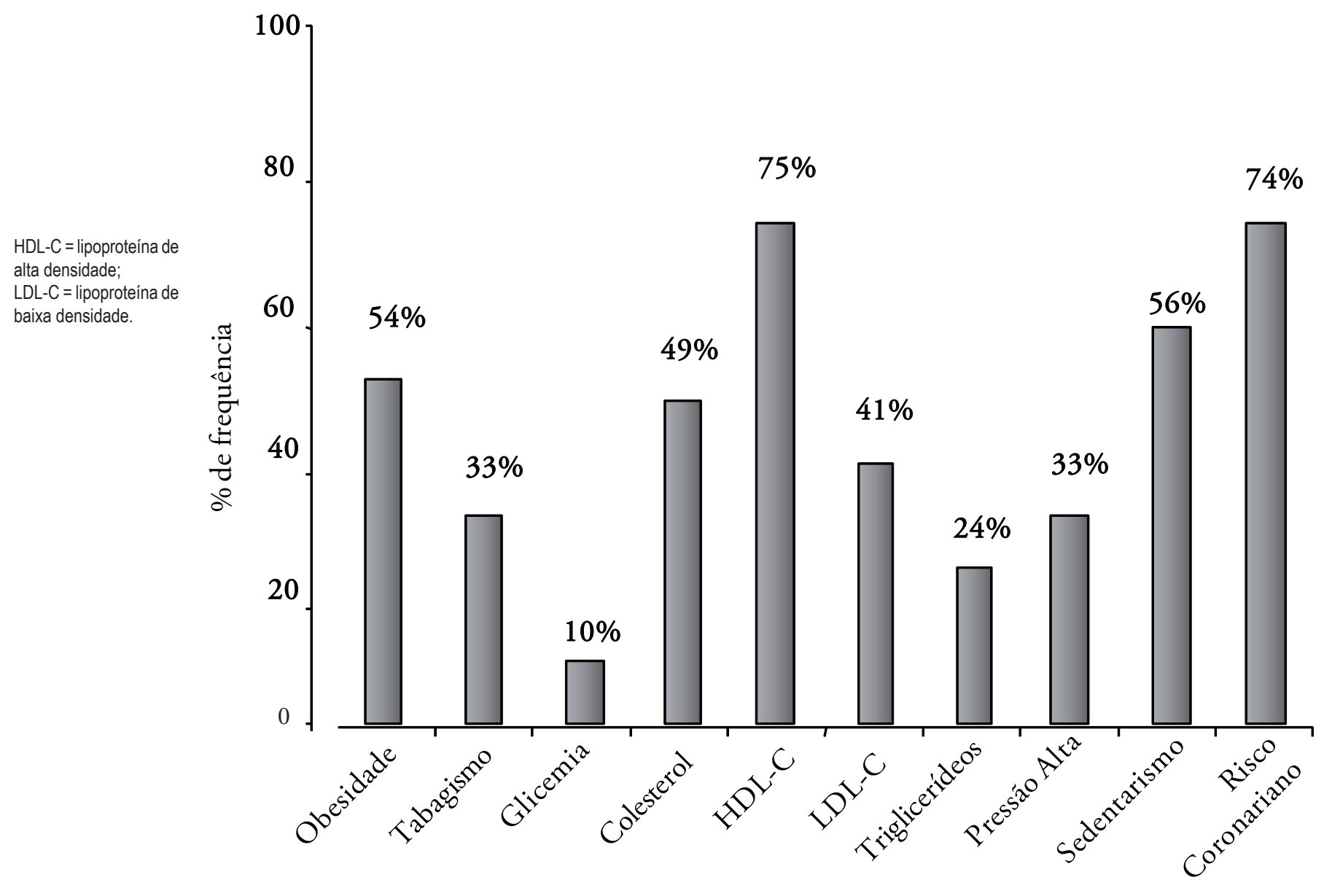

FIGURA 1 - Distribuição da frequência dos fatores de risco dos funcionários da saúde.

TABELA 1 - Distribuição de frequência das variáveis estudadas segundo classificação e sexo, com respectivos valores de qui-quadrado.

HDL-C = lipoproteína de

alta densidade;

LDL-C = lipoproteína de

baixa densidade.

PAS = Pressão arterial

sistólica.

\begin{tabular}{|c|c|c|c|c|}
\hline \multirow{2}{*}{ Variável } & \multirow{2}{*}{ Classificação } & \multicolumn{2}{|c|}{ Sexo } & \multirow{2}{*}{$X^{2}(p)$} \\
\hline & & Feminino & Masculino & \\
\hline \multirow[t]{2}{*}{ Obesidade } & $20-25 \mathrm{~kg} / \mathrm{m}^{2}$ & 66 & 18 & \\
\hline & $25-30 \mathrm{~kg} / \mathrm{m}^{2}$ & 47 & 14 & $0,31(\mathrm{p}>0,05)$ \\
\hline \multirow[t]{3}{*}{ Tabagismo } & Nunca Fumou & 103 & 25 & \\
\hline & Ex-fumante & 23 & 9 & $1,26(\mathrm{p}>0,05)$ \\
\hline & Fumante & 25 & 8 & \\
\hline Glicemia & $70-110 \mathrm{mg} / \mathrm{dL}$ & 134 & 29 & \\
\hline \multirow[t]{3}{*}{ Colesterol } & $<200 \mathrm{mg} / \mathrm{dL}$ & 69 & 15 & \\
\hline & $200-239 \mathrm{mg} / \mathrm{dL}$ & 52 & 14 & $0,95(\mathrm{p}>0,05)$ \\
\hline & > $239 \mathrm{mg} / \mathrm{dL}$ & 24 & 8 & \\
\hline
\end{tabular}


TABELA 1 - Distribuição de frequência das variáveis estudadas segundo classificação e sexo, com respectivos valores de qui-quadrado (continuação).

\begin{tabular}{|c|c|c|c|c|}
\hline \multirow{2}{*}{ Variável } & \multirow{2}{*}{ Classificação } & \multicolumn{2}{|c|}{ Sexo } & \multirow{2}{*}{$\mathbf{X}^{2}(\mathbf{p})$} \\
\hline & & Feminino & Masculino & \\
\hline \multirow[t]{3}{*}{ HDL-C } & $>60 \mathrm{mg} / \mathrm{dL}$ & 26 & 2 & \multirow{3}{*}{$25,17(\mathrm{p}<0,001)$} \\
\hline & $40-60 \mathrm{mg} / \mathrm{dL}$ & 98 & 15 & \\
\hline & $<40 \mathrm{mg} / \mathrm{dL}$ & 18 & 18 & \\
\hline \multirow[t]{3}{*}{ LDL-C } & $<129 \mathrm{mg} / \mathrm{dL}$ & 72 & 14 & \multirow{3}{*}{$1,69(\mathrm{p}>0,05)$} \\
\hline & $130-159 \mathrm{mg} / \mathrm{dL}$ & 44 & 11 & \\
\hline & $>159 \mathrm{mg} / \mathrm{dL}$ & 21 & 5 & \\
\hline \multirow[t]{3}{*}{ Triglicerídeos } & $<150 \mathrm{mg} / \mathrm{dL}$ & 111 & 20 & \multirow{3}{*}{$11,43(\mathrm{p}<0,01)$} \\
\hline & $150-200 \mathrm{mg} / \mathrm{dL}$ & 19 & 5 & \\
\hline & $>200 \mathrm{mg} / \mathrm{dL}$ & 13 & 11 & \\
\hline \multirow[t]{3}{*}{ PAS } & $<130 \mathrm{mmHg}$ & 103 & 20 & \multirow{3}{*}{$8,90(\mathrm{p}<0,05)$} \\
\hline & $130-139 \mathrm{mmHg}$ & 17 & 12 & \\
\hline & > $139 \mathrm{mmHg}$ & 28 & 9 & \\
\hline \multirow[t]{3}{*}{ Sedentarismo } & Não & 52 & 17 & \multirow{3}{*}{$1,24(\mathrm{p}>0,05)$} \\
\hline & Sim & 91 & 20 & \\
\hline & Baixo & 57 & 16 & \\
\hline \multirow[t]{2}{*}{ Fatores de risco } & Moderado & 54 & 13 & \multirow{2}{*}{$0,69(\mathrm{p}>0,05)$} \\
\hline & Elevado & 4 & 2 & \\
\hline
\end{tabular}

TABELA 2 - Taxa de “odds” das variáveis estudadas segundo sexo.

\begin{tabular}{lccc}
\hline \multicolumn{1}{c}{$\begin{array}{c}\text { Variável } \\
\text { (Valor de corte/Risco) }\end{array}$} & \multicolumn{2}{c}{ Sexo } & Intervalo de confiança \\
\cline { 2 - 3 } & Feminino & Masculino & $(0,50-2,01)$ \\
\hline Sobrepeso/obesidade $\left(>25 \mathrm{~kg} / \mathrm{m}^{2}\right)$ & 1,00 & 1,00 & $(0,72-2,95)$ \\
Tabagismo (ex-fumante/fumante) & 1,00 & 1,45 & $(0,97-7,43)$ \\
Glicemia $(>110 \mathrm{mg} / \mathrm{dL})$ & 1,00 & 2,69 & $(0,63-2,77)$ \\
Colesterol Total $(>200 \mathrm{mg} / \mathrm{dL})$ & 1,00 & 1,33 & $(0,57-11,62)$ \\
HDL-C $(<60 \mathrm{mg} / \mathrm{dL})$ & 1,00 & 2,57 & $(0,57-2,79)$ \\
LDL-C $(>129 \mathrm{mg} / \mathrm{dL})$ & 1,00 & 1,26 & $(1,28-5,96)$ \\
Triglicerídeos $(>150 \mathrm{mg} / \mathrm{dL})$ & 1,00 & 2,77 & $(1,18-4,86)$ \\
Hipertensão $($ PAS $>130 \mathrm{mmHg})$ & 1,00 & 2,40 & $(0,71-3,08)$ \\
Sedentarismo (presente) & 1,00 & 1,48 & $(0,54-5,20)$ \\
Risco coronariano (baixo/moderado/alto) & 1,00 & 1,68 & \\
\hline
\end{tabular}

HDL-C = lipoproteína de alta densidade; LDL-C = lipoproteína de baixa densidade.

$$
\text { baixa densidade. }
$$

A FIGURA 2 (A e C) apresenta a distribuição percentual da capacidade respiratória $\left(\mathrm{VO}_{2}\right)$ dos funcionários da saúde segundo sexo. Os valores de $\mathrm{VO}_{2}$ máximo, considerados como ruins foram observados em $56 \%$ dos homens e $23 \%$ das mulheres. A condição de normalidade foi obtida por $9 \%$ dos homens e $24 \%$ das mulheres. Por outro lado, os valores de $\mathrm{VO}_{2}$ máximo acima da normalidade foram, observados em
35 e $53 \%$ dos homens e mulheres, respectivamente. A análise de qui-quadrado confirmou que a condição cardiovascular ruim no sexo masculino apresentava taxa significativamente superior a do feminino $(p<0,01)$.

A FIGURA 2 ( $\mathrm{B}$ e D) informa os valores percentuais da flexibilidade dos funcionários da saúde segundo sexo. Baixo nível de flexibilidade foi observado em $80 \%$ dos homens e $76 \%$ das mulheres. Valores limítrofes foram
HDL-C = lipoproteína de alta densidade; LDL-C = lipoproteína de baixa densidade. PAS = Pressão arterial sistólica. 
encontrados em $10 \%$ dos homens e $9 \%$ das mulheres, somente $10 \%$ dos homens e $15 \%$ das mulheres apresentaram bons níveis de flexibilidade. Esses dados apontam que a maioria dos funcionários apresenta valores insatisfatórios de flexibilidade.

Casos de obesidade foram registrados em $48 \%$ dos homens e $75 \%$ das mulheres. As taxas de massa gorda identificadas como normais para a faixa etária estudada foi de $17 \%$ para o sexo masculino e apenas $9 \%$ para o feminino. Entre os funcionários classificados com uma boa porcentagem de gordura, observou-se $35 \%$ para homens e $16 \%$ para mulheres (dados não demonstrados). Apesar destas discrepâncias, o teste de qui-quadrado e a taxa de "odds" não identificaram diferenças significativas entre os sexos para a composição corporal (TABELA 2).
A

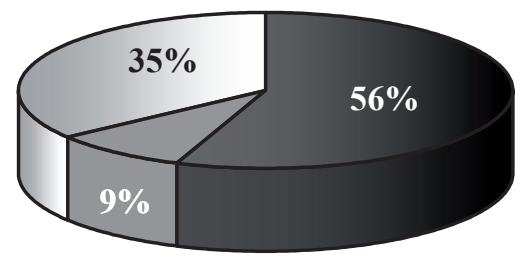

$>39 \mathrm{ml} / \mathrm{kg} / \mathrm{min}$

$37-39 \mathrm{ml} / \mathrm{kg} / \mathrm{min}$

$<37 \mathrm{ml} / \mathrm{kg} / \mathrm{min}$

C

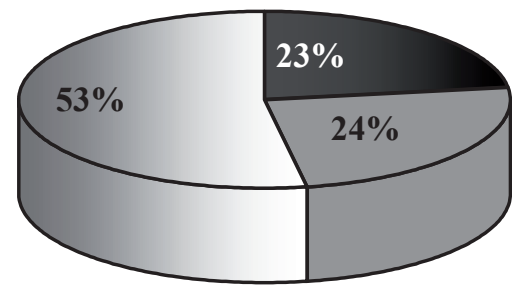

$>31 \mathrm{ml} / \mathrm{kg} / \mathrm{min}$

$26-31 \mathrm{ml} / \mathrm{kg} / \mathrm{min}$

$<26 \mathrm{ml} / \mathrm{kg} / \mathrm{min}$
Homens

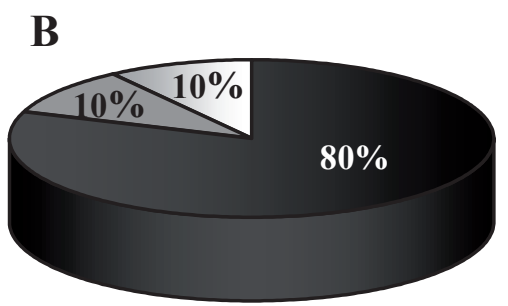

$>28 \mathrm{~cm}$

$24-28 \mathrm{~cm}$

$<24 \mathrm{~cm}$

Mulheres

D

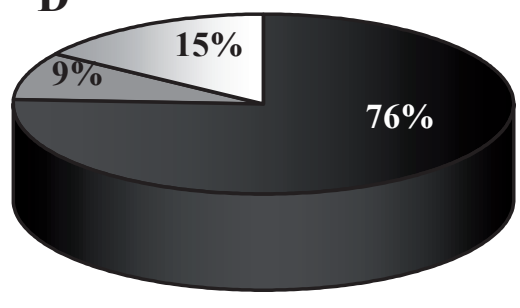

$\begin{aligned}> & 33 \mathrm{~cm} \\ & 30-33 \mathrm{~cm} \\ < & 30 \mathrm{~cm}\end{aligned}$

FIGURA 2 - Distribuição da frequência da capacidade cardiorrespiratória (painel da esquerda, A e C) e da flexibilidade (painel da direita, B e D) dos funcionários da saúde.

A TABELA 3 representa os resultados das correlações entre variáveis hemodinâmicas, antropométricas e bioquímicas. Pode-se observar que o IMC se correlacionou positivamente com PA (sistólica e média), CT, TG e glicemia, tanto para mulheres como para homens. O IMC contribui negativamente para os valores de flexibilidade somente das mulheres, ou seja, quanto maior o IMC das mulheres, menor sua flexibilidade. Constatou-se também que, para as mulheres, a idade é fator agravante para as variáveis hemodinâmicas (PAS, PAD e PAM), antropométricas (IMC), bioquímicas (CT, LDL-C, glicemia exceto TG). O aumento da idade não acarreta alteraçôes nas variáveis analisadas dos homens. 
TABELA 3 - Correlação entre parâmetros hemodinâmicos, antropométricos e bioquímicos segundo sexo.

\begin{tabular}{llcccc}
\hline \multirow{2}{*}{ Variável } & & \multicolumn{2}{c}{ Feminino } & \multicolumn{2}{c}{ Masculino } \\
\cline { 3 - 5 } IMC & & $\mathbf{r}$ & valor de $\mathbf{p}$ & $\mathbf{r}$ & valor de $\mathbf{p}$ \\
\cline { 3 - 5 } & PA Sistólica & 0,3094 & 0,0001 & 0,4221 & 0,0060 \\
& PA Diastólica & 0,2686 & 0,0011 & 0,2181 & 0,1708 \\
& PA Média & 0,2991 & 0,0002 & 0,3115 & 0,0474 \\
& Colesterol & 0,2520 & 0,0026 & 0,3987 & 0,0160 \\
& Triglicerídeos & 0,4581 & 0,0001 & 0,5629 & 0,0004 \\
Glicemia & 0,2305 & 0,0058 & 0,7457 & 0,0000 \\
Flexibilidade & 0,2695 & 0,0009 & 0,0155 & 0,9226 \\
& LDL-C & 0,1856 & 0,0318 & 0,0373 & 0,8477 \\
& & & & 0,6655 \\
& PA Sistólica & 0,4037 & 0,0001 & 0,0696 & 0,3356 \\
PA Diastólica & 0,2682 & 0,0010 & 0,1543 & 0,4332 \\
PA Média & 0,3678 & 0,0001 & 0,1258 & 0,8080 \\
& IMC & 0,3141 & 0,0001 & 0,0392 & 0,1098 \\
Flexibilidade & 0,2633 & 0,0012 & 0,2535 & 0,9995 \\
Colesterol & 0,3846 & 0,0001 & 0,0001 & 0,9383 \\
Triglicerídeos & 0,1461 & 0,0838 & 0,0131 & 0,6355 \\
LDL-C & 0,3558 & 0,0001 & 0,0919 & 0,5040 \\
Glicemia & 0,2401 & 0,0037 & 0,1168 & \\
\hline
\end{tabular}

IMC = índice de massa corporal;

$\mathrm{PA}=$ pressão arterial;

LDL-C = lipoproteína de

baixa densidade

\section{Discussão}

Uma das principais observações deste trabalho foi que a condição física dos servidores da saúde necessita de cuidados. Pode-se observar que grande parcela dos avaliados apresentava capacidade cardiorrespiratória ruim, com porcentagem de gordura acima do esperado, índices de flexibilidade insatisfatórios e taxas elevadas de sedentarismo.

A presença de um ou mais fatores de risco, como observado neste estudo, demonstra que esta população tem maior probabilidade de ser acometida por agravos cardiovasculares (Gus, FisCHMANN \& MEDINA, 2002; Hwang, Tsai \& Chen, 2006; KIVIMÄKI, LeINO-ARJAS, LUUKKONEN, RIIHIMÄKI, VAHTERA \& KIRJONEN, 2002; Rosini, Machado \& Xavier, 2006; Viebig et al., 2006), principalmente quando associado ao estilo de vida sedentário (Elley, KerSe \& Arroll, 2003; Feio, Fonseca, Rego, Feio, Elias, Costa, Izar, Paola \& Carvalho, 2003; Gal, Santos \& Barros, 2005; MaTOS et al., 2004; VIEBIG et al., 2006). O reconhecimento destes fatores faz-se importante, uma vez que até o ano de 2006, último levantamento do Datasus, 34\% e 32\% dos moradores de Bauru e Jaú, respectivamente, morreram por problemas cardiovasculares (BRASIL, 2009).
Apesar dos benefícios do exercício físico serem amplamente reconhecidos (AMARAL, Zorn \& MIChelini, 2000; Kelley, Kelley \& Frankling, 2005; Pescatello, Franklin, Fagard, Farquhar, Kelley $\&$ RAY, 2004), 56\% dos servidores investigados neste estudo são sedentários, concordando com resultados de outros autores em importantes cidades do Brasil (Gus, Fischmann \& Medina, 2002; Matos et al., 2004). GaL, Santos e Barros (2005) encontraram na população da cidade do Porto, Portugal, porcentagens ainda maiores de sedentarismo, valores estes que atingiram $84 \%$ dos avaliados. Estas taxas são semelhantes às médias nacionais (SOCIEDADE BRASIleira de CARDiologia, 2005). Por outro lado, Viebig et al. (2006) demonstraram que somente 4-6\% dos pacientes de uma clínica médica de São Paulo, uma das principais cidades do Brasil, são sedentários. Esta discrepância pode estar associada ao critério para determinar o nível de atividade física entre os estudos.

A análise do perfil lipídico do presente estudo mostrou que, aproximadamente metade dos servidores apresenta taxas de CT e LDL-C acima do esperado. Os valores de HDL-C também estão 
abaixo da faixa de normalidade para $75 \%$ dos funcionários. Estas porcentagens incorporam todos os indivíduos que se encontram dentro da faixa limítrofe para cada variável. Estes resultados são semelhantes aos constatados por MATOS et al. (2004) nos centros de pesquisa da Petrobrás, e na cidade de Brusque, Santa Catarina (Rosini, Machado \& XAVIER, 2006). Quando são consideradas somente as taxas mais elevadas (CT $>240 \mathrm{mg} / \mathrm{dL}$, LDL-C > $160 \mathrm{mg} / \mathrm{dL}$ e TG > $200 \mathrm{mg} / \mathrm{dL}$ ), os dados observados no presente estudo são menores do que outros da literatura (MATOS et al., 2004; VALLES et al., 1997). Os valores de CT obtidos nesta investigação estão abaixo da média nacional (Pollock \& Wilmore, 1993), no entanto, as taxas de TG são concordantes (Sociedade Brasileira de Cardiologia, 2005).

Apesar dos valores das variáveis bioquímicas não estarem significativamente elevados, eles se correlacionam positivamente (na maioria dos casos) com o índice de massa corporal, tanto para homens como para mulheres. Considerando que $54 \%$ dos servidores da pesquisa apresentam peso corporal acima da normalidade, sugere-se que alguma medida preventiva com relação IMC seja adotada para evitar complicações futuras no perfil lipídico. Adicionalmente, observou-se também, que os valores de CT e LDL-C foram correlacionados com a idade, pelo menos das mulheres, indicando que, se medidas preventivas forem implementadas precocemente, maiores serão as possibilidades de se obter resultados satisfatórios. Importante ressaltar também que a redução dos valores de CT pode contribuir para reduzir os outros fatores de risco associados a ele, tais como: LDL-C e TG.

A prevalência de diabetes no presente estudo foi de $4 \%$ (valores acima de $126 \mathrm{mg} / \mathrm{dL}$ ), semelhante à encontrada por FoRJAZ, TINUCCI, BARTHOLOMEU, Fernandes, Casagrande e Massucato (2002). Quando as taxas limítrofes de glicemia foram consideradas (entre 110 e $126 \mathrm{mg} / \mathrm{dL}$ ), a porcentagem aumentou para $10 \%$, semelhante à média nacional para a mesma faixa etária (SOCIEDADE BRASILEIRA DE CARdiologia, 2005), porém, maior que a encontrada em outros estudos (Gus et al., 2004; MATOS et al., 2004; Rosini, Machado \& Xavier, 2006; VAlles et al., 1997; Viebig et al., 2006). Hwang, Tsai e Chen (2006) revelaram que 70,8\% dos trabalhadores obesos da área hospitalar apresentavam diabetes, sugerindo expressiva correlação entre estas variáveis que foi também observada na presente pesquisa, tanto em homens quanto em mulheres. De acordo com Chunang, Chou, Hsu, Cheng, Tsai, Lin e CHEn (2006), obesidade, associada ou não com diabetes, é um importante fator de risco para doenças cardiovasculares.

Sobrepeso e obesidade são condiçôes clínicas complexas que envolvem múltiplos fatores e caracterizam-se pela deposição de gordura decorrente de alterações metabólicas, endócrinas e comportamentais. Os resultados observados nesta investigação não permitem determinar a causa da obesidade ( $23 \%$ dos casos), mas pode-se pressupor que esteja correlacionada, predominantemente, com alteraçôes metabólicas e comportamentais, pois mais de $40 \%$ dos servidores apresentaram problemas no perfil lipídico e 56\% eram sedentários. Neste sentido, o IMC se correlacionou com a maioria das variáveis hemodinâmicas e bioquímicas em ambos os sexos. O importante é que, isoladamente, a obesidade e sua progressão, contribuem significativamente para o desenvolvimento de hipertensão arterial (CHUANG et al., 2006), que tem sido considerada um grande problema de saúde pública (CHOBANian, BaKris, Black, Cushman, Green, Izzo Junior, Jones, Materson, Oparil, Wright Junior \& Roccella, 2003). Quando associada à resistência à insulina, idade, álcool e microalbuminúria, a obesidade aumenta ainda mais as chances de hipertensão arterial (Chuang et al., 2006; Wang, Lee, Fabsitz, Devereux, Best, Welty \& Howard, 2006). Frente a estes resultados, medidas de mudanças comportamentais devem ser estimuladas, uma vez que a redução de 9-13\% do peso corporal pode contribuir de forma expressiva para reduzir $6 \mathrm{mmHg}$ a PAS e aproximadamente $4 \mathrm{mmHg}$ a PAD (WINNICKI, BONSO, Dorigatti, Longo, Zaetta, Mattarei, D’Este, Laurini, Pessina \& Palatini, 2006).

Adicionalmente, observou-se que o risco de hipertensão arterial nos homens foi 2,4 vezes maior que nas mulheres, o que pode ser explicado devido à grande participação do sistema renina angiotensina no sexo masculino, como demonstrado por Reckelhoff, Zhang e Srivastava (2000). Apesar de nem todos os estudos epidemiológicos apontarem o sexo como precursor de hipertensão arterial (Chuang et al., 2006; Ong, Cheung, Man, Lau \& KAREN, 2007), é importante ressaltar que $21 \%$ dos homens avaliados neste estudo apresentaram níveis de PAS > $140 \mathrm{mmHg}$, o que significa risco de mortalidade 6,2 vezes maior do que aqueles que apresentam PAS < $127 \mathrm{mmHg}$ (KIVIMÄKI et al., 2002). Esta alta prevalência de HA nos homens foi observada independentemente da idade, ou seja, mesmo em idades menos avançadas a presença de HA já era observada. 
O processo de envelhecimento também contribui significativamente no aumento da pressão arterial, principalmente quando associado a outros fatores, tais como, resistência à insulina e obesidade (Chuang et al., 2006; Ong et al., 2007). No presente estudo, o aumento da idade se correlacionou positivamente com PAS, PAD e PAM somente nas mulheres. Sobre estes resultados, recentes estudos têm observado associaçõos entre incremento do peso corporal (VIEBIG et al., 2006) e pressão arterial (ONG et al., 2007) com o avançar dos anos, sendo mais prevalente nas faixas etárias dos 45 aos 65 anos de idade, momento em que ocorre o período de pós menopausa, caracterizado por grandes alterações metabólicas e cardiovasculares nas mulheres. Este pode ser um dos motivos pelo qual não foram encontradas correlações entre a idade e PA, IMC, flexibilidade, colesterol e glicemia entre os homens, pois a faixa etária dos homens é menor que a das mulheres.

$\mathrm{O}_{2}$ máximo, utilizado como indicador de aptidão cardiorrespiratória, demonstrou que mais da metade dos homens e $1 / 4$ das mulheres apresentaram condição debilitada nas avaliações. Da mesma forma, os índices de flexibilidade foram menores que os normais tanto para homens $(80 \%)$ quanto para mulheres $(76 \%)$. Estes resultados apontam que ambos os sexos caracterizaram-se como grupo de risco para perda de autonomia por distúrbios do aparelho locomotor e cardiovascular. Estes dados, associados com a alta prevalência de obesidade, sugerem tratar-se de população de hábitos sedentários e com condição nutricional desbalanceada, o que contribui significativamente para aumentar o risco de doenças cardiovasculares (KIVIMÄKI et al., 2002).

Por outro lado, tem sido demonstrado que o estilo de vida ativo nem sempre resulta em proteção para o risco de doenças cardiovasculares. Estudo realizado por Hu, Tuomilehto, Silventoinen, BARENGO e Jousilahti (2004), na Finlândia, constatou que os níveis pressóricos não apresentaram correlação com a condição física dos avaliados. Estes resultados indicam que a prevenção de doenças cardiovasculares é muito mais complexa do que pode parecer. O efeito protetor contra as doenças cardiovasculares depende de um conjunto de açōes que devem ser conduzidas por diferentes profissionais de saúde, cada qual atuando de acordo com sua competência.

A coexistência de múltiplos fatores de risco nestes servidores demonstra tratar-se de população não assistida, o que implica em possíveis causas de aposentadorias precoces. Como estes indivíduos passam aproximadamente 40 horas semanais no trabalho, medidas preventivas deveriam ser incentivadas, visando mudanças no estilo de vida. Orientações nutricionais, consultas médicas anuais e sessões de alongamento/exercício físico podem auxiliar efetivamente para alterar hábitos nocivos à saúde. Considerando que a população analisada é madura, com boa escolaridade e a maioria encontra-se na faixa limítrofe dos parâmetros avaliados, uma intervenção multiprofissional, neste momento, poderia contribuir efetivamente para evitar que os processos crônicos observados possam evoluir.

\section{Abstract}

Association of cardiovascular disease risk factors and quality of life among health workers

Several public health problems are due to environmental work, which, associated with sedentarism, contribute to reduce the quality of life of the worker. Therefore, this study aimed to describe the profile, evaluate the physical condition and cardiovascular risk factor of health workers. Health workers (198) from Bauru and Jau cities ( $46 \pm 10$ years old) were assessed. They answered a questionnaire and participated in antrophometric and functional assessments. Values are presented as means \pm SD. Qui-squared $\left(x^{2}\right)$ analysis, odds rate and Pearson correlation were used $(\mathrm{p}<0.05)$. From all workers evaluated, 54\% presented high body weight, 33\% high blood pressure and 56\% were sedentary. Total cholesterol (TC), low-density lipoprotein and triglycerides (TG) above normal values were found in $49 \%, 41 \%$ and $24 \%$ of the individuals, respectively, and $75 \%$ presented low values of high-density lipoprotein. Qui-squared analysis and odds rate confirmed that TG and blood pressure (BP) were higher for men compared with women. Physical capacity was bad for 56\% of men and $23 \%$ of women. Also, flexibility was unsatisfactory for men and women. Body mass index correlated positively with $\mathrm{BP}, \mathrm{TC}$ and TG. Aging was an important risk factor for BP, TC, blood glucose, body mass index and flexibility only for women $(p<0.05)$. The prevalence of multiple risk factors among these health workers shows that 
this population is not adequately assisted. For this reason, a program from the government in order to make this population recognize such problems and avoid premature retirement is suggested.

UnITERMS: Hypertension; Diabetes; Sedentarism; Obesity.

\section{Referências}

AMARAL, S.L.; ZORN, T.M.T.; MICHELINI, L.C. Exercise training normalizes wall-to-lumen ratio of the gracilis muscle arterioles and reduces pressure in spontaneously hypertensive rats. Journal of Hypertension, London, v. 18, p. 1563-72, 2000. BRASIL. Ministério da Saúde. Departamento de Informática do SUS. Datasus. Disponível em: <http://w3.datasus.gov. br/datasus/datasus.php>. Acesso em: 17 mar. 2009.

CHOBANIAN, A.V.; BAKRIS, G.L.; BLACK, H.R.; CUSHMAN, W.C.; GREEN, L.A.; IZZO JUNIOR, J.L.; JONES, D.W.; MATERSON, B.J.; OPARIL, S.; WRIGHT JUNIOR, J.T.; ROCCELLA, E.J. Joint National Committee on Prevention, Detection, Evaluation, and Treatment of High Blood Pressure. National Heart, Lung, and Blood Institute; National High Blood Pressure Education Program Coordinating Committee. Seventh report of the Joint National Committee on Prevention, Detection, Evaluation, and Treatment of High Blood Pressure. Hypertension, Dallas, v.42, p.1206-52, 2003. CHUANG, S.Y.; CHOU, P.; HSU, P.F.; CHENG, H.M.; TSAI, S.T.; LIN, I.F.; CHEN, C.H. Presence and progression of abdominal obesity are predictors of future high blood pressure and hypertension. American Journal of Hypertension, New York, v.19, p.788-95, 2006.

ELLEY, C.R.; KERSE, N.M.; ARROLL, B. Why target sedentary adults in primary health care? Baseline results from the Waikato heart, health, and activity study. Preventive Medicine, New York, v.37, p.342-8, 2003.

FEIO, C.M.A.; FONSECA, F.A.H.; REGO, S.S.; FEIO, M.N.B.; ELIAS, M.C.; COSTA, E.A.S.; IZAR, M.C.O.; PAOLA, A.A.V.; CARVALHO, A.C.C. Lipid profile and vardiovascular tisk in two Amazonian populations. Arquivos Brasileiros de Cardiologia, São Paulo, v.81, p.596-9, 2003.

FORJAZ, C.L.M.; TINUCCI, T.; BARTHOLOMEU, T.; FERNANDES, T.E.M.; CASAGRANDE, V.; MASSUCATO J.G. Assessment of the cardiovascular risk and physical activity of individuals exercising at a public park in the City of São Paulo. Arquivos Brasileiros de Cardiologia, São Paulo, v.79, p.35-42, 2002.

GAL, D.L.; SANTOS, A.C.; BARROS, H. Leisure-time versus full-day energy expenditure: a cross-sectional study of sedentarism in a Portuguese urban population. BioMed Central Public Health, London, v.5, p.1-7, 2005.

GUS, I.; FISCHMANN, A.; MEDINA, C. Prevalência dos fatores de risco da doença arterial coronariana no Estado do Rio Grande do Sul. Arquivos Brasileiros de Cardiologia, São Paulo, v.78, p.478-83, 2002.

GUS, I.; HARZHEIM, E.; ZASLAVSKY, C.; MEDINA, C.; GUS, M. Prevalence, awareness, and control of systemic arterial hypertension in the State Rio Grande do Sul. Arquivos Brasileiros de Cardiologia, São Paulo, v.83, p.429-33, 2004. HU, G.; TUOMILEHTO, J.; SILVENTOINEN, K.; BARENGO, N.; JOUSILAHTI, P. Joint effects of physical activity, body mass index, waist circumference and waist-to-hip ratio with the risk of cardiovascular disease among middle-aged finnish men and women. European Heart Journal, London, v.25, p.2212-9, 2004.

HWANG, L.C.; TSAI, C.H.; CHEN, T.H. Overweight and obesity-related metabolic disorders in hosital employees. Journal of the Formosan Medical Association, Taipei, v.105, p.56-63, 2006.

KAVEY, R.E.W.; DANIELS, S.R.; LAUER, R.M.; ATKINS, D.L.; HAYMAN, L.L.; TAUBERT, K. American Heart Association guidelines for primary prevention of atherosclerotic cardiovascular disease beginning in childhood. Circulation, Dallas, v.107, p.1562-6, 2003.

KELLEY, G.A.; KELLEY, K.S.Z.; FRANKLING, B. Tran aerobic exercise, lipids and lipoproteins in overweight and obese adults: a meta-analysis of randomized controlled trials. International Journal of Obesity, London, v.29, p.881-93, 2005. KIVIMÄKI, M.; LEINO-ARJAS, P.; LUUKKONEN, R.; RIIHIMÄKI, H.; VAHTERA, J.; KIRJONEN, J. Work stress and risk of cardiovascular mortality: prospective cohort study of industrial employees. British Medical Journal, London, v.325, p.1-5, 2002.

MATOS, M.F.D.; SILVA, N.A.S.; PIMENTA, A.J.M.; CUNHA, A.J.L.A. Prevalence of risk factors for cardiovascular disease in employess of the research center at Petrobras. Arquivos Brasileiros de Cardiologia, São Paulo, v.82, p. 5-8, 2004. ONG, K.L.; CHEUNG, B.M.Y.; MAN, Y.B.; LAU, C.P.; KAREN, S.L. Lam prevalence, awareness, treatment, and Control of hypertension among United States adults 1999-2004. Hypertension, Dallas, v.49, p.69-75, 2007.

302 • Rev. bras. Educ. Fís. Esporte, São Paulo, v.24, n.2, p.293-303, abr./jun. 2010 
PESCATELLO, L.S.; FRANKLIN, B.A.; FAGARD, R.; FARQUHAR, W.B.; KELLEY, G.A.; RAY, C.A. American College of Sports Medicine Position Stand. Exercise and Hypertension. Medicine \& Science in Sports \& Exercise, Madison, v.36, p.533-53, 2004.

POLLOCK, M.I.; WILMORE, J.H. Exercícios na saúde e na doença: avaliação e prescrição para prevenção e rehabilitação. 2.ed. São Paulo: Medsi, 1993. p.1-736.

RECKELHOFF, J.F.; ZHANG, H.; SRIVASTAVA, K. Gender differences in development of hypertension in spontaneously hypertensive rats role of the renin-angiotensin system. Hypertension, Dallas, v.35, part 2, p.480-3, 2000.

ROSINI, N.; MACHADO, M.J.; XAVIER, H.T. Study of the prevalence and multiplicity of cardiovascular risk factors in hypertensive individuals from the city of Brusque, SC, Brazil. Arquivos Brasileiros de Cardiologia, Brasil, v.86, p.219-22, 2006.

SOCIEDADE BRASILEIRA DE CARDIOLOGIA. Atlas: corações do Brasil. São Paulo: SBC, 2005. v.1, p.40-54.

IV Diretrizes de dislipidemias e prevenção de aterosclerose. São Paulo: SBC, 2007. v.10.

SOCIEDADE BRASILEIRA DE CARDIOLOGIA. SOCIEDADE BRASILEIRA DE HIPERTENSÃO. SOCIEDADE BRASILEIRA DE NEFROLOGIA. V Diretrizes Brasileiras de Hipertensão Arterial. Revista Brasileira de Hipertensão, Ribeirão Preto, v.13, n.4, p.256-312, 2006.

STUMER, T.; HASSELBACH, P.; AMELANG, M. Personality, lifestyle, and risk of cardiovascular disease and cancer: follow-up of population based cohort. British Medical Journal, London, v.10, n.332, p.1359, 2006.

THOMPSON, P.D.; BUCHNER, D.; PINA, I.L.; BALADY, G.J.; WILLIAMS, M.A.; MARCUS, B.H.; BERRA, K.; BLAIR, S.N.; COSTA, F.; FRANKLIN, B.; FLETCHER, G.F.; GORDON, N.F.; PATE, R.R.; RODRIGUEZ, B.L.; YANCEY, A.K.; WENGER, N.K. Exercise and physical activity in the prevention and treatment of atherosclerotic cardiovascular disease. Circulation, Dallas, v.107, p.3109-16, 2003.

VALLES, M.; MATE, G.; BRONSOMS, J.; CAMPINS, M.; ROSELLO, J.; TORQUET, P.;MAURI, J.M. Prevalence of arterial hypertension and other cardiovascular risk factors among hospital workers. Medicina Clinica, Barcelona, v.108, p.613-4, 1997.

VIEBIG, R.F.; VALERO, M.P.; ARAÚJO, F.; YAMADA, A.T.; MANSUR, A.J. Cardiovascular health profile of an adult population from the Metropolitan of São Paulo. Arquivos Brasileiros de Cardiologia, São Paulo, v.86, p.353-60, 2006. WANG, W.; LEE, E.T.; FABSITZ, R.R.; DEVEREUX, R.; BEST, G.L.; WELTY, T.K.; HOWARD, B.V. A longitudinal study of hypertension risk factors and their relation to cardiovascular disease the strong heart study. Hypertension, Dallas, v.47, p.403-9, 2006.

WINNICKI, M.; BONSO, E.; DORIGATTI, F.; LONGO, D.; ZAETTA, V.; MATTAREI M.; D’ESTE, D.; LAURINI, G.; PESSINA, A.C.; PALATINI, P. Effect of body weight loss on blood pressure after 6 years of follow-up in stage 1 hypertension. American Journal of Hypertension, New York, v.19, p.1103-9, 2006.

\section{Agradecimentos}

Os autores agradecem ao Sr. Amilton Alves Teixeira, Diretor da DRS-VI Bauru e a Sra. Vera Helena Perlatti D’Alpino, responsável pelo NGA-25 Jaú, por suas contribuições no desenvolvimento deste estudo, incentivo para o programa e avaliações médicas. Os autores agradecem também aos monitores do LAPE pelo auxílio nas avaliações dos funcionários.

Sandra Lia do Amaral

Departamento de Educação Física Universidade Estadual Paulista - Campus Bauru Av. Eng. Luis Edmundo Carrijo Coube, 14-01 17033-360 - Bauru - SP - BRASIL e-mail: slamaral@fc.unesp.br

Recebido para publicação: 27/03/2009

1a. Revisão: 16/09/2009

2a. Revisão: 23/11/2009

Aceito: 14/12/2009 